Portland State University

PDXScholar

\title{
Automated Traffic and the Finite Size Resonance
}

J. J. P. Veerman

Portland State University, veerman@pdx.edu

Borko D. Stošić

Universidade Federal Rural de Pernambuco

F. M. Tangerman

Follow this and additional works at: https://pdxscholar.library.pdx.edu/mth_fac

Part of the Control Theory Commons

Let us know how access to this document benefits you.

\section{Citation Details}

Veerman, J. J. P.; Stošić, Borko D.; and Tangerman, F. M., "Automated Traffic and the Finite Size Resonance" (2009). Mathematics and Statistics Faculty Publications and Presentations. 153.

https://pdxscholar.library.pdx.edu/mth_fac/153

This Post-Print is brought to you for free and open access. It has been accepted for inclusion in Mathematics and Statistics Faculty Publications and Presentations by an authorized administrator of PDXScholar. Please contact us if we can make this document more accessible: pdxscholar@pdx.edu. 


\title{
Automated Traffic and The Finite Size Resonance
}

\author{
J. J. P. Veerman*(1,2), B. D. Stošić (3), F. M. Tangerman (4), \\ (1) Dept. of Math. \& Stat., Portland State University, Portland, OR 97201, USA. \\ (2) Dip. Ing. Inf. \& Mat. App., Univ. di Salerno, Salerno, Italy. \\ (3) Dept. Estad. \& Inf., Univ. Federal Rural de Pernambuco, 52171-900, Recife-PE, Brazil. \\ (4) Northport, NY.
}

May 31, 2008

\begin{abstract}
We investigate in detail what one might call the canonical (automated) traffic problem: A long string of $N+1$ cars (numbered from 0 to $N$ ) moves along a one-lane road "in formation" at a constant velocity and with a unit distance between successive cars. Each car monitors the relative velocity and position of only its neighboring cars. This information is then fed back to its own engine which decelerates (brakes) or accelerates according to the information it receives. The question is: What happens when due to an external influence - a traffic light turning green — the 'zero'th' car (the "leader") accelerates?

As a first approximation, we analyze linear(ized) equations and show that in this scenario the traffic flow has a tendency to be stop-and-go. We give approximate solutions for the global traffic as function of all the relevant parameters (the feed back parameters as well as cruise velocity and so on). We discuss general design principles for these algorithms, that is: how does the choice of parameters influence the performance.
\end{abstract}

\section{Introduction}

The movement of collections of agents (or 'flocks') with a single leader (and a directed path from it to every agent) can be stabilized over time as has been shown before (for details see [6] and prior references therein, shorter descriptions are given in $[3,9]$ ). But for large flocks, even when this is the case, perturbations in the movement of the leader nonetheless may grow to a considerable size as they propagate throughout the flock and before they die out over time. This is due (in the setting of this study) to a curious resonance phenomenon which we discussed in [8] and is related to the existence of an intrinsic response latency (or finite response time) over finite distances. We dubbed this phenomenon the "finite size resonance" because it is caused by near cancelation of various terms of a denominator and it occurs precisely when the number of agents is large but finite.

In this paper we apply these ideas to study in detail what one might call the canonical (automated) traffic problem: A long string of $N+1$ agents (numbered from 0 to $N$ ) moves along a one-lane road "in formation" (or coherently, see [6]) at a constant velocity and with a prescribed distance between successive cars. Each car monitors the relative velocity and position of its immediate neighbors (in front and behind it). This information is fed back to its own engine which decelerates (brakes) or accelerates according to the information it receives (see Equation (2.1)). The question is: What happens when due to an external influence - a traffic light turning green or red for example — the 'zero'th' car (the "leader") accelerates or decelerates? Traffic flow problems such as these have been studied intensively using different methods. For example at a macroscopic level, one can express the conservation of number of vehicles in terms of the vehicle density, leading to a Partial Differential Equation exhibiting shock waves (see [5]). In this paper we are interested in a description at the so-called microscopic level where each car is modeled individually. At this level cellular automata are sometimes used to study traffic (see [10]). However in this work the motion of each car is modeled by a linear (second order) Ordinary Differential Equation coupled to the motion of certain nearby cars.

*e-mail: veerman@pdx.edu 
Most models considered in the literature emphasize the coupling exclusively to the car directly in front ([7], [2], [11]) and are generally called Follow-the-Leader models.

As an example of this consider the following instance of a Follow-the-Leader model (see [7]):

$$
x_{k}^{\prime \prime}=C \frac{x_{k-1}^{\prime}-x_{k}^{\prime}}{x_{k-1}-x_{k}} .
$$

( $C$ is a constant.) Linearize around a solution where all agents travel at the same velocity and where the distance between successive cars equals 1 :

$$
x_{k}(t)=-k+v_{0} \cdot t+\epsilon \xi_{k}
$$

The in formation motion (ie: $\epsilon=0$ ) is a solution. Furthermore the first order expansion in $\epsilon$ gives a system that corresponds to the look forward system discussed in Section 3 of [8] but with $f=0$. This system is not stable since the eigenvalue 0 has large multiplicity. This is related to the fact that Equation 1.2 for $\epsilon=1$ and $\xi_{k}$ arbitrary constants, is an $N$ dimensional family of solutions of Equation (1.1). If in a dense traffic situation we want to insure that the spacing between cars is indeed asymptotically equal to 1 , we may choose $f<0$. We are then in the situation of Corollary 1 of [8], where oscillations grow exponentially as they propagate from one car to the next. If on the other hand one decides to look at a model similar to Equation (2.1) but with the relative weight of the car in the back equal to $\rho$ and of the car in front equal to $1-\rho$ then any perturbation is also amplified exponentially as it propagates except when $\rho=1 / 2$, the case dealt with here. These assertions are not straightforward and will be shown in forthcoming work.

In this work we exhibit the asymptotic (as the number of agents increases) solution of the canonical traffic problem mentioned earlier. If the system is stabilized and the leader executes a harmonic motion $e^{i \omega t}$, then the motion of the last car can be written as $a_{N}(\omega) e^{i \omega t}$. We calculate the response function $a_{N}$ (Lemma 3.2). The response function is then approximated by a pole expansion (Theorem 4.2). This allows us to express the motion of the trailing car in terms of the eigenvalues of the system given in equation (2.1) (see Corollary 4.3) as well as in terms of those of the Laplacian operator given in Section 2 that describes the interaction between cars (see Equation 4.3). In the last Section we discuss qualitative properties of the solution, and the influence of the parameters on the solution.

We expect the phenomena studied here also to be relevant in the case of movements of large flocks in two or three dimensions (birds, for example). In these cases we expect higher dimensional versions of the wave-like phenomena described here to occur in flocks that are close to 'in formation' flight (see for example [12]).

\section{Acknowledgements}

We thank Arturo Olvera and the referee for several useful remarks. JJPV gratefully acknowledges grants through the Dip. Ing. Inf. \& Mat. App., Univ. di Salerno, Salerno, Italy, and the Ist. 'Mauro Piccone' per le Applicazioni del Calcolo, Rome, Italy, that made an extended stay at these institutions possible, as well as the generous hospitality offered by these institutions.

\section{The Model}

While any likely algorithm for designing automated traffic would almost certainly be nonlinear, nonetheless the linearization of the equations around an in formation solution would be a subject of study.

Let us suppose that the flock (ie: the collection of agents) is traveling along the line and that the individual agents have positions $x_{i}$ in the real line where $i \in\{0, \cdots N\}$. Agent $i$ adjusts its acceleration according to a preprogrammed algorithm considering (with equal weight) the positions and velocities of its 'neighbors': one agent in front and one agent behind him. The zeroth agent (or 'leader'), however, does not pay attention to the agent behind it ( and no-one is in front) and simply accelerates or decelerates according, for example, to the traffic lights it encounters. The model is given by:

$$
\forall i \in\{1, \cdots N-1\}: \dot{x}_{i}=u_{i}
$$




$$
\begin{aligned}
\dot{u}_{i} & =f\left\{\left(x_{i}-h_{i}\right)-\frac{1}{2}\left(x_{i-1}-h_{i-1}+x_{i+1}-h_{i+1}\right)\right\}+g\left\{u_{i}-\frac{1}{2}\left(u_{i-1}+u_{i+1}\right)\right\} \\
\dot{x}_{N} & =u_{N} \\
\dot{u}_{N} & =f\left\{\left(x_{N}-h_{N}\right)-\left(x_{N-1}-h_{N-1}\right)\right\}+g\left\{u_{N}-u_{N-1}\right\} \\
\text { and } \quad x_{0} & =x_{0}(t) \text { given }
\end{aligned}
$$

The parameters $h_{i}$ determine the desired relative distances between agents $i$ and $i-1$ as $h_{i}-h_{i-1}$. These feedback parameters $f$ and $g$ are independent of $i$ and time.

It is advantageous to write these equations in a more compact form. Following [6], Section 6, we introduce $x \equiv\left(x_{1}, u_{1}, x_{2}, u_{2}, \cdots, x_{N}, u_{N}\right)^{T}$. Now let $h \equiv\left(h_{1}, 0, h_{2}, 0 \cdots, h_{N}, 0\right)^{T}$. Use the Kronecker product $(\otimes)$ to write the equations of motion (2.1)) with a single independent leader more succinctly as a first order system:

$$
\dot{x}=I_{N} \otimes A x+P \otimes K(x-h)+L_{0} \otimes\left(K\left(\begin{array}{c}
x_{0}(t)-h_{0} \\
u_{0}(t)
\end{array}\right)\right) .
$$

Here $I_{N}$ is the $N$-dimensional identity. The $N$ dimensional matrix $P$ and the $N$ vector $L_{0}$ are obtained from the Directed Laplacian $L$ given by

$$
L=\left(\begin{array}{cccccc}
0 & 0 & 0 & 0 & \cdots & \cdots \\
-1 / 2 & 1 & -1 / 2 & 0 & \cdots & \cdots \\
0 & -1 / 2 & 1 & -1 / 2 & \cdots & \cdots \\
\vdots & & & & & \\
\cdots & \cdots & \cdots & 0 & -1 & 1
\end{array}\right)
$$

$P$ is obtained by removing the first row and column (corresponding to the leader) from $L$ :

$$
P=\left(\begin{array}{cccccc}
1 & -1 / 2 & 0 & 0 & \ldots & \ldots \\
-1 / 2 & 1 & -1 / 2 & 0 & \ldots & \ldots \\
0 & -1 / 2 & 1 & -1 / 2 & \cdots & \cdots \\
\vdots & & & & & \\
\cdots & \ldots & \ldots & 0 & -1 & 1
\end{array}\right)
$$

The vector $L_{0}$ consists of the last $N$ entries of the first (the leader's) column of $L$, and $A$ and $K$ are given by

$$
A \equiv\left(\begin{array}{cc}
0 & 1 \\
0 & 0
\end{array}\right) \quad \text { and } \quad K \equiv\left(\begin{array}{cc}
0 & 0 \\
f & g
\end{array}\right) \text {. }
$$

Substituting $z=x-h$ one obtains:

$$
\dot{z}=\left(I_{N} \otimes A+P \otimes K\right) z+\Gamma(t):=M z+\Gamma(t) \quad,
$$

where $\Gamma(t)$ represents the forcing term in Equation (2.2). Since $z=0$ corresponds to an in formation solution, the (real part of the) eigenvalues of $M$ determine whether this in formation motion is stable.

\section{Preliminary Results}

To insure that the in formation solution is stable, the eigenvalues of $M$ must have negative real part. The spectrum of the matrix $P$ is given by (see for example the exercises on pages 216-218 of [13]):

$$
\bigcup_{\ell \in\{0, \cdots N-1\}}\left\{\lambda_{\ell}\right\} \equiv \bigcup_{\ell \in\{0, \cdots N-1\}}\left\{1-\cos \left(\frac{2 \ell+1}{2 N} \pi\right)\right\} .
$$


According to $[3,6,9]$ the eigenvalues of $M$ are given by the solutions $\nu_{\ell \pm}$ of

$$
\nu^{2}-\lambda_{\ell} g \nu-\lambda_{\ell} f=0 \quad \Longrightarrow \quad \nu_{\ell \pm}=\frac{1}{2}\left(\lambda_{\ell} g \pm \sqrt{\left(\lambda_{\ell} g\right)^{2}+4 \lambda_{\ell} f}\right) \quad,
$$

where $\lambda_{\ell}$ runs through the spectrum of $P$.

Lemma 3.1 i): The system is stabilized if and only if both $f$ and $g$ are strictly smaller than zero.

ii): Given $K>0$ and $f$ and $g$ negative. Fix $\ell_{0}$ is the largest integer less than $K N^{1 / 2}$. For $N$ large enough, the eigenvalues with smallest (in modulus) real part are simple and given by:

$$
\ell \leq \ell_{0} \quad \text { and } \quad \Re \nu_{\ell \pm}=-\frac{(2 \ell+1)^{2} \pi^{2}|g|}{16 N^{2}}+\mathcal{O}\left(N^{-4}\right) \quad \text { and } \quad \Im \nu_{\ell \pm}= \pm \frac{(2 \ell+1) \pi \sqrt{|f|}}{2 \sqrt{2} N}+\mathcal{O}\left(N^{-3}\right)
$$

Proof: The first of these statements follows from $\lambda_{i}>0$ and Equation (3.2).

For the second statement, note that if $\frac{2|f|}{g^{2}} \geq 1$, the argument of the root in Equation (3.2) is always negative. The real part of $\nu_{\ell \pm}$ equals $\frac{1}{2} \lambda_{\ell} g$ and the imaginary part, $\pm \frac{1}{2} \sqrt{\left(\lambda_{\ell} g\right)^{2}+4 \lambda_{\ell} f}$.

If on the other hand $\frac{2|f|}{g^{2}}<1$, we proceed as follows. Note $0<\lambda_{0}<\cdots \lambda_{N-1}<2$. Define

$$
\ell_{1} \equiv \min _{\ell \in\{0, \cdots N-1\}}\left\{\ell \mid \lambda_{\ell}>\frac{4|f|}{g^{2}}\right\} .
$$

By Equation (3.1), if $N$ is large enough then

$$
\frac{4|f|}{g^{2}}\left(1-\sqrt{1-\frac{2|f|}{g^{2}}}\right)>\lambda_{\ell_{0}}
$$

Thus we have (if $N$ is large enough):

$$
\begin{array}{ll}
\ell \geq \ell_{1} \quad: \quad \Re \nu_{\ell \pm} \leq \frac{1}{2} \lambda_{\ell} g\left(1-\sqrt{1-\frac{4|f|}{\lambda_{\ell} g^{2}}}\right)<\frac{1}{2} \lambda_{\ell_{1}} g\left(1-\sqrt{1-\frac{2|f|}{g^{2}}}\right)<\frac{1}{2} \lambda_{\ell_{0}} g \\
\ell<\ell_{0} \quad: \quad \Re \nu_{\ell \pm}=\frac{1}{2} \lambda_{\ell} g>\frac{1}{2} \lambda_{\ell_{0}} g
\end{array}
$$

Thus $\Re \nu_{\left\{\ell \pm, \ell \geq \ell_{1}\right\}}<\Re \nu_{\left\{\ell \pm, \ell<\ell_{0}\right\}}$ which implies the statement.

Suppose the leader sustains a harmonic motion $e^{i \omega t}$ and the asymptotic orbit of the $k$-th agent is $a_{k}(\omega) e^{i \omega t}$. The complex-valued function $a_{k}$ is called the frequency response (of the $k$-th agent).

Remark: We will from now on always assume that $f$ and $g$ are negative.

Lemma 3.2 The frequency response function of the $k$-th agent is given by

$$
\begin{gathered}
a_{k}(f, g, \omega)=\frac{\mu_{-}^{N-k}+\mu_{+}^{N-k}}{\mu_{-}^{N}+\mu_{+}^{N}} \\
\text { where } \mu_{ \pm} \equiv \gamma \pm \sqrt{\gamma^{2}-1} \quad \text { and } \quad \gamma=\frac{f+i \omega g+\omega^{2}}{f+i \omega g}
\end{gathered}
$$

Proof: The full details are in [8]. Assume the system of equations (2.3) is stabilized so that all eigenvalues of $M$ have negative real part. Let $z_{0}(t)$ be given by $e^{i \omega t}$. Under these hypotheses the motion of the system is asymptotic (as $t \rightarrow \infty)$ to $z_{k}=a_{k} \cdot e^{i \omega t}$. This leads to a recursive equation on the $a_{k}$,

$$
a_{k-1}-2 \gamma a_{k}+a_{k+1}=0 \text {. }
$$


Let $\mu_{ \pm}$be the roots of the associated characteristic polynomial $P(x)=1-2 \gamma x+x^{2}$. The boundary conditions are given by:

$$
a_{0}=1 \quad \text { and } \quad-a_{N-1}+\gamma a_{N}=0 \quad .
$$

The general solution is $a_{k}=c_{-} \mu_{-}^{k}+c_{+} \mu_{+}^{k}$. A convenient way to solve for $c_{ \pm}$is by setting $d_{1}=c_{-} \mu_{-}^{N}$ and $d_{2}=c_{+} \mu_{+}^{N}$. The boundary conditions can be rewritten as

$$
\left(\begin{array}{cc}
\mu_{-}^{-N} & \mu_{+}^{-N} \\
\gamma-\mu_{-}^{-1} & \gamma-\mu_{+}^{-1}
\end{array}\right)\left(\begin{array}{l}
d_{1} \\
d_{2}
\end{array}\right)=\left(\begin{array}{l}
1 \\
0
\end{array}\right) .
$$

Use the characteristic polynomial to show that

$$
\gamma-\mu_{ \pm}^{-1}=\frac{\mu_{ \pm}-\mu_{ \pm}^{-1}}{2}
$$

Since $\mu_{ \pm}^{-1}=\mu_{\mp}$, one obtains (after dividing the second row by a common factor):

$$
\left(\begin{array}{cc}
\mu_{+}^{N} & \mu_{-}^{N} \\
1 & -1
\end{array}\right)\left(\begin{array}{l}
d_{1} \\
d_{2}
\end{array}\right)=\left(\begin{array}{l}
1 \\
0
\end{array}\right) .
$$

Denote this last matrix by $M$; the same matrix with the first row replaced by ones, by $M^{*}$. Then it is easy to see that

$$
a_{N}=d_{1}+d_{2}=\operatorname{det} M^{*} / \operatorname{det} M
$$

which gives the desired answer. The other $a_{k}$ are found from the general solution.

Near cancelation in the denominator of $a_{N}$ occurs when $\left|\mu_{ \pm} e^{ \pm \frac{i \pi}{2 N}}\right|=\mathcal{O}\left(N^{-2}\right)$ causing $a_{N}$ to be large. This indeed happens for certain $\omega$ close to zero, and this is called 'near resonance', or 'resonance' for short. We end the section by quoting a last result from [8].

Theorem 3.3 For large flocks (near) resonance occurs at $\omega=\omega_{N}$ where

$$
\omega_{N}=\frac{\sqrt{|f|} \pi}{2 \sqrt{2} N}+\mathcal{O}\left(N^{-3}\right)
$$

and its peak size is given by

$$
a_{N}\left(\omega_{N}\right)=\frac{8 \sqrt{2|f|}}{\pi^{2}|g|} N+\mathcal{O}\left(N^{-1}\right)
$$

\section{Main Results}

The leader's motion is given by:

$$
\ddot{z}_{0}(t)=a_{0}\left(H(t)-H\left(\frac{v_{0}}{a_{0}}-t\right)\right) \quad, \quad \dot{z}_{0}(0)=0 \quad, \quad x_{0}(0)=0
$$

where $H$ is the Heaviside function.

The fact that the Fourier transform of $z_{0}$ diverges is a problem. To circumvent this, consider the second derivative of the equations of motion (2.1). Set $y_{i} \equiv \ddot{x}_{i}=\ddot{z}_{i}$. We have:

$$
\begin{aligned}
\forall i \in\{1, \cdots N-1\}: \ddot{y}_{i} & =f\left\{y_{i}-\frac{1}{2}\left(y_{i-1}+y_{i+1}\right)\right\}+g\left\{\dot{y}_{i}-\frac{1}{2}\left(\dot{y}_{i-1}+\dot{y}_{i+1}\right)\right\} \\
\ddot{y}_{N} & =f\left\{y_{N}-y_{N-1}\right\}+g\left\{\dot{y}_{N}-\dot{y}_{N-1}\right\} \\
\text { and } \quad y_{0} & =a_{0}\left(H(t)-H\left(\frac{v_{0}}{a_{0}}-t\right)\right)
\end{aligned}
$$


The Fourier Transform $q$ of $\ddot{z}_{0}(t)$ is:

$$
q(\omega) \equiv a_{0} \int_{0}^{v_{0} / a_{0}} e^{-i \omega t} d t \quad \text { where } \quad \ddot{x}_{0}(t)=\frac{1}{2 \pi} \int q(\omega) e^{i \omega t} d \omega .
$$

The strategy is to solve this system and then integrate twice and add the appropiate Galilean motion. (In $\mathbb{R}$ a Galilean motion is a motion of the form $x(t)=v_{0} t+x_{0}$ where $x_{0}$ and $v_{0}$ are constants.)

Proposition 4.1 Let $a_{k}$ be as given in Lemma 3.2. Then

$$
y_{k}=\frac{1}{2 \pi} \int_{\mathbb{R}} a_{k}(\omega) q(\omega) e^{i \omega t} d \omega .
$$

solves Equation (4.1) including boundary conditions.

Proof: The $a_{k}$ satisfy

$$
\begin{gathered}
a_{k-1}-2 \gamma a_{k}+a_{k+1}=0 \quad, \\
a_{0}=1 \quad \text { and } \quad-a_{N-1}+\gamma a_{N}=0 \quad .
\end{gathered}
$$

Using differentiation under the integral sign and linearity of the integral, one sees that this implies that the $y=$ $\left(y_{1}(t), \dot{y}_{1}(t), y_{2}(t), \cdots, \dot{y}_{N}(t)\right)^{T}$ solves Equation (4.1) including boundary conditions.
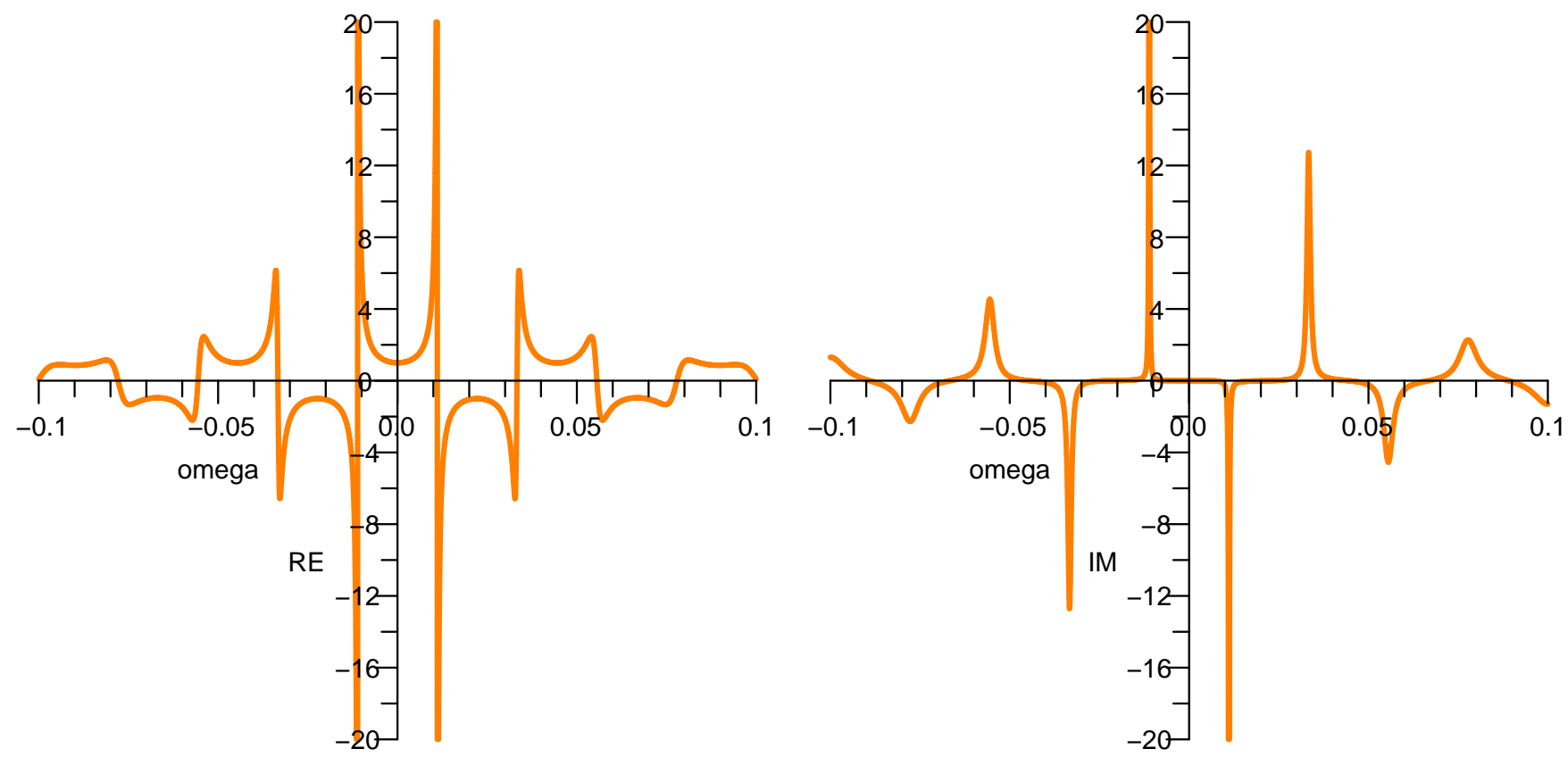

Figure 4.1: A close-up of the real and imaginary parts of $a_{N}(\omega)$ for $f=g=-1$.

The graph of the function $a_{N}(\omega)$ has a number of large $\mathcal{O}(N)$ peaks (see Figure 4.1). However, given some small $\epsilon>0$, then for $N$ large enough its modulus is greater than $\epsilon$ only when $|\omega|<L N^{-1 / 2}$ for some $L$ (see Appendix). It can be approximated using a pole expansion involving $\mathcal{O}\left(N^{-1 / 2}\right)$ terms (see Figure 4.2).

Theorem 4.2 Let $\nu_{ \pm}$as in $\ell_{0}$ as in Lemma 3.1. Then for $N$ large enough, the first $\ell_{0}$ terms of the pole expansion of $a_{N}(\omega)$ are given by:

$$
a_{N}(\omega)=\frac{\sqrt{|f|}}{N \sqrt{2}} \sum_{\ell=0}^{\ell_{0}}(-1)^{\ell}\left(\frac{1}{\omega+i \nu_{\ell-}}-\frac{1}{\omega+i \nu_{\ell+}}\right) .
$$




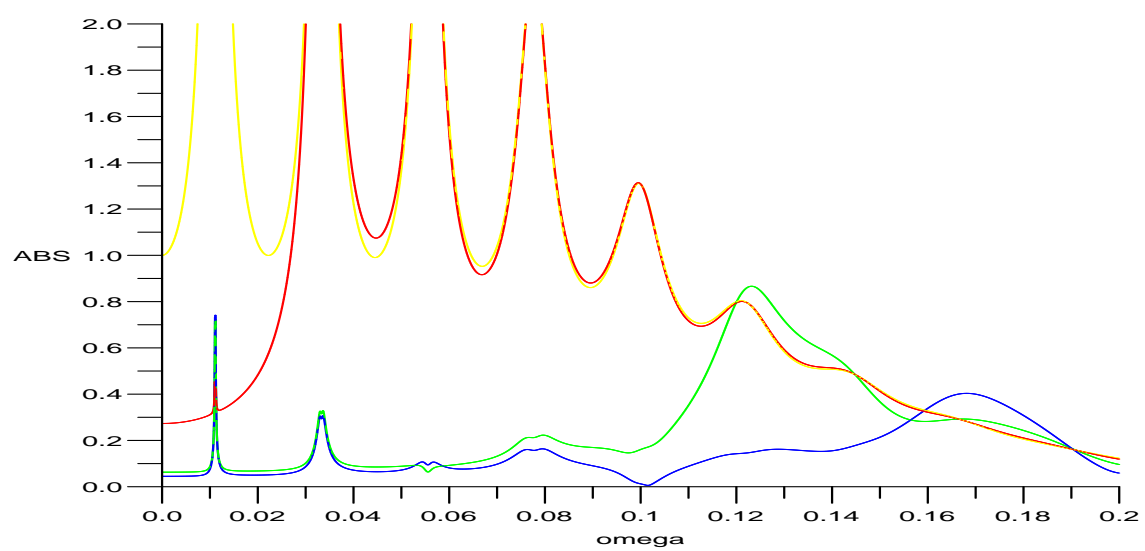

Figure 4.2: The parameters in this picture are $N=100, f=g=-1 . \quad$ Yellow: $\left|a_{N}\right|$, red: $\left|a_{100}-\sum_{\ell=0}^{0}\left(\frac{\operatorname{Res}_{\ell-}}{\omega+i \nu_{\ell-}}+\frac{\operatorname{Res}_{\ell+}}{\omega+i \nu_{\ell+}}\right)\right|$, green: $\left|a_{100}-\sum_{\ell=0}^{4}\left(\frac{\operatorname{Res}_{\ell-}}{\omega+i \nu_{\ell-}}+\frac{\operatorname{Res}_{\ell+}}{\omega+i \nu_{\ell+}}\right)\right|$, blue: $\left|a_{100}-\sum_{\ell=0}^{6}\left(\frac{\operatorname{Res}_{\ell-}}{\omega+i \nu_{\ell-}}+\frac{\operatorname{Res}_{\ell+}}{\omega+i \nu_{\ell+}}\right)\right|$.

Proof: We assume that $N$ is large enough so that Lemma 3.1 holds.

First note that (see Lemma 3.2) $a_{N}(\omega)=\frac{2}{\mu_{+}^{N}+\mu_{-}^{N}}$. Substitute the appropriate expression in $\gamma$ for $\mu_{ \pm}$and use the binomial theorem to see that all odd powers of $\sqrt{\gamma^{2}-1}$ cancel. Thus $a_{N}$ is a rational function of $\gamma$ and therefore also of $\omega$. The same considerations show that the denominator of this rational function has lower degree than the numerator.

The poles of $a_{N}$ are given exactly by $-i \nu_{\ell \pm}$ where $\nu_{\ell \pm}$ are the eigenvalues of $M$ - see Equations (2.3) and (3.2). It is clear from these equations that the eigenvalues are simple. Thus

$$
a_{N}(\omega)=\sum_{\ell=0}^{N}\left(\frac{\operatorname{Res}_{\ell-}\left(a_{N}(\omega)\right)}{\omega+i \nu_{\ell-}}+\frac{\operatorname{Res}_{\ell+}\left(a_{N}(\omega)\right)}{\omega+i \nu_{\ell+}}\right),
$$

where $\operatorname{Res}_{\ell-} a_{N}(\omega)$ are the residues of $a_{N}(\omega)$. Approximate $a_{N}$ using $\ell_{0}$ poles associated to the eigenvalues with the smallest real part (see Lemma 3.1):

$$
a_{N}(\omega) \approx \sum_{\ell=0}^{\ell_{0}}\left(\frac{\operatorname{Res}_{\ell-} a_{N}(\omega)}{\omega+i \nu_{\ell-}}+\frac{\operatorname{Res}_{\ell+} a_{N}(\omega)}{\omega+i \nu_{\ell+}}\right),
$$

To calculate the residues, note that $a_{N}(\omega)$ is the composition of

$$
a_{N}\left(\mu_{+}\right)=\frac{2 \mu_{+}^{N}}{\mu_{+}^{2 N}+1}
$$

with $\mu_{+}=\mu_{+}(\omega)$. We will drop the subscript ' + ' from $\mu_{+}$for the remainder of this proof. $a_{N}(\mu)$ has $2 N$ poles at $\mu_{\ell \pm} \equiv e^{i \frac{2 \ell+1}{2 N} \pi}$ for $\ell$ in $\{0, \cdots, N-1\}$. Thus these poles are in 1-1 correspondence to another. From the expansion of $\mu$ (see $[8]): \mu(\omega)=1+i \frac{\sqrt{2}}{\sqrt{|f|}} \omega$ and the order $1 / N$ expansion of $-i \nu_{\ell \pm}$ (see Lemma 3.1), one sees that:

$$
\mu\left(-i \nu_{\ell \pm}\right)=e^{ \pm i \frac{2 \ell+1}{2 N} \pi}
$$

Note that at a pole $-i \nu_{\ell \pm}$, the residue is given by

$$
\operatorname{Res}_{\ell \pm} a_{N}(\mu)=\lim _{\mu \rightarrow \mu_{\ell \pm}}\left(\mu-\mu_{\ell \pm}\right) a_{N}(\mu)=\mu^{\prime}\left(-i \nu_{\ell \pm}\right) \lim _{\omega \rightarrow-i \nu_{\ell \pm}}\left(\omega+i \nu_{\ell \pm}\right) a_{N}(\mu(\omega))=\mu^{\prime}\left(-i \nu_{\ell \pm}\right) \operatorname{Res}_{\ell \pm} a_{N}(\omega)
$$


On the other hand, the residues of Equation (4.2) can be calculated as the numerator divided by the derivative of the denominator evaluated at the pole:

$$
\operatorname{Res}_{\ell \pm} a_{N}(\mu)=\frac{2 \mu_{\ell \pm}^{N}}{2 N \mu_{\ell \pm}^{2 N-1}}=\frac{1}{N} \mu_{\ell \pm}^{1-N}
$$

From the Taylor expansion of $\mu(\omega)$ :

$$
\mu^{\prime}\left(-i \nu_{\ell \pm}\right)=i \frac{\sqrt{2}}{\sqrt{|f|}}+\mathcal{O}\left(\nu_{\ell}\right)=i \frac{\sqrt{2}}{\sqrt{|f|}} \mu_{\ell \pm}+\mathcal{O}\left(\nu_{\ell}\right)
$$

Putting all this together gives:

$$
\operatorname{Res}_{\ell \pm} a_{N}(\omega)=\frac{\sqrt{|f|}}{N \sqrt{2}} \frac{\mu_{\ell \pm}^{-N}}{i}=\mp(-1)^{\ell} \frac{\sqrt{|f|}}{N \sqrt{2}}
$$

which proves the result.

For the motion of the last car we now obtain the following corollary.

Corollary 4.3 Given $a_{0}$ and $v_{0}$ and taking into account only low frequency (high amplitude) contributions of $a_{N}(\omega)$ (see Appendix), then as $N$ tends to infinity, the orbit of the trailing agent tends to:

$$
x_{N}(t)=c_{0}+v_{0} t-i v_{0} \sum_{\ell=0}^{\ell_{0}} \frac{\operatorname{Res}_{\ell-} a_{N}(\omega)}{\left(\Im \nu_{\ell-}\right)^{2}}\left(e^{\nu_{\ell-} t}-e^{\nu_{\ell+} t}\right) .
$$

where $x_{N}(0)$ and $v_{0}$ are integration constants determined by the motion of the leader.

Proof: From Proposition 4.1:

$$
\ddot{x}_{N}(t)=\frac{1}{2 \pi} \int_{\mathbb{R}} a_{k}(\omega) q(\omega) e^{i \omega t} d \omega
$$

Theorem 4.2 allows us to replace this with

$$
\ddot{x}_{N}(t)=\frac{1}{2 \pi} \sum_{\ell=0}^{\ell_{0}} \operatorname{Res}_{\ell_{-}} a_{N}(\omega) \int_{\mathbb{R}}\left(\frac{1}{\omega+i \nu_{\ell-}}-\frac{1}{\omega+i \nu_{\ell+}}\right) q(\omega) e^{i \omega t} d \omega .
$$

The function $q$ is defined just prior to Proposition 4.1 and satisfies

$$
q(\omega)=v_{0}\left(1+\mathcal{O}\left(\omega v_{0} / a_{0}\right)\right) .
$$

Since we take $|\omega|<L N^{-1 / 2}$ we can replace $q$ by the constant $v_{0}$. Now $\Re \nu_{\ell \pm}<0$ implies

$$
\frac{1}{2 \pi} \int_{\mathbb{R}} \frac{e^{i \omega t}}{\omega+i \nu_{\ell \pm}} d \omega=i H(t) e^{\nu_{\ell \pm} t}
$$

substituting this into the expression for $\ddot{x}_{N}$, one obtains:

$$
\ddot{x}_{N}(t)=i v_{0} \sum_{\ell=0}^{\ell_{0}} \operatorname{Res}_{\ell-} a_{N}(\omega)\left(e^{\nu_{\ell_{-}} t}-e^{\nu_{\ell_{+}} t}\right)
$$

From Lemma 3.1 we recall that up to order $N^{-1}, \nu_{\ell \pm}^{-2}$ equals $-\left(\Im \nu_{\ell-}\right)^{-2}$. Twice integrating the expression for $\ddot{x}_{N}$ then yields the result (the two constants of integration give rise to the Galilean motion $c_{0}+v_{0} t$ ).

Therefore we can express the motion almost completely in terms of the eigenvalues of the Laplacian $L$ (where $H$ is the Heaviside function):

$$
x_{N}(t)=\left(c_{0}+v_{0} t+2 v_{0} \sum_{\ell=0}^{\ell_{0}} \frac{\operatorname{Res}_{\ell-} a_{N}(\omega)}{\lambda_{\ell}^{2} g^{2}+4 \lambda_{\ell} f} e^{-\frac{\lambda_{\ell} g}{2}} \sin \left(\sqrt{\left(\lambda_{\ell}^{2} g^{2}+4 \lambda_{\ell} f\right)} t\right)\right) H(t)
$$




\section{Discussion of Results}

We begin by expressing the motion given in Corollary 4.3 in terms of the parameters (dropping the Heaviside $H(t)$ from the notation).

$$
x_{N}(t)=-\frac{8 \sqrt{2}}{\pi^{2}|f|^{1 / 2}} N v_{0} \sum_{\ell=0}^{\ell_{0}} \frac{(-1)^{\ell} e^{-\frac{(2 \ell+1)^{2} \pi^{2}|g|}{16 N^{2}} t} \sin \left((2 \ell+1) \frac{\pi|f|^{1 / 2}}{2 \sqrt{2} N} t\right)}{(2 \ell+1)^{2}}+\text { Galilean. }
$$

In continuation we discuss some particulars of this solution (see Figure 5.1).
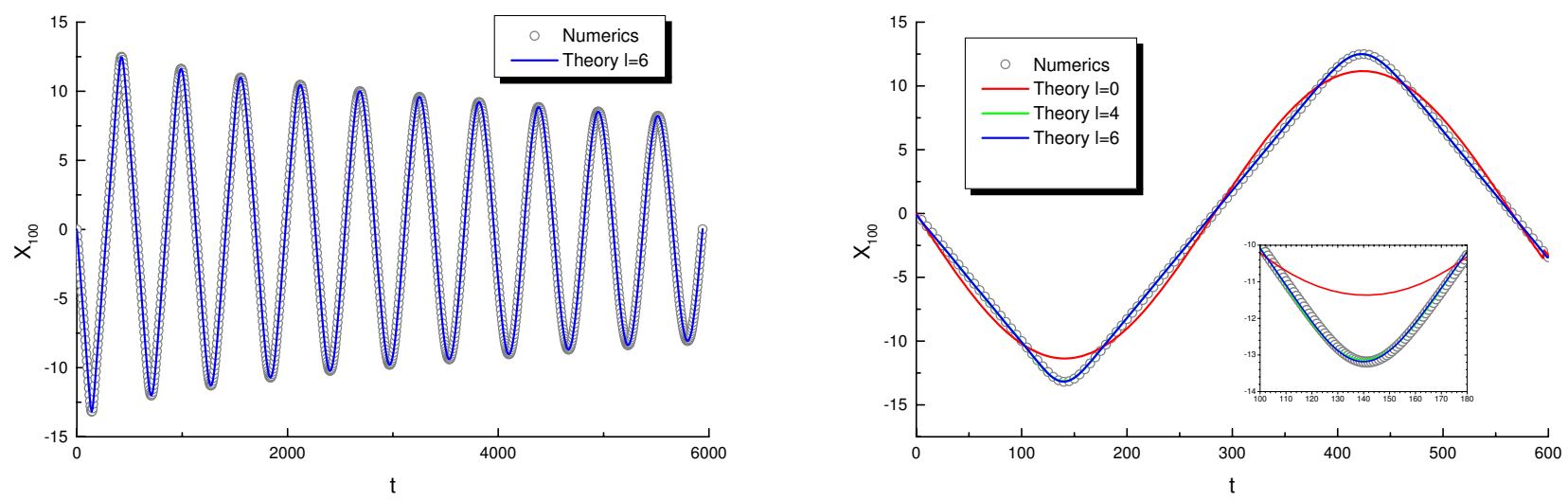

Figure 5.1: The $\ell_{0}=6$ approximation in Equation (5.1) (with $f=g=-1, N=100, v_{0}=0.1$, and $a_{0}=10$ ) coincides almost perfectly with the numerics. We have plotted $x_{N}(t)-x_{0}(t)$ in the case. The right figure is a magnification of the left.

After the leader starts moving, there is a response time $T_{r}$ (entirely due to inertia). More precisely: the response time $T_{r}=\frac{\sqrt{2}}{|f|^{1 / 2}} N$ is a quarter of the fundamental period $\frac{2 \pi}{\omega_{N}}$. Car $k$ will have a response time that equals $k / N$ times that of the last car. The wave velocity or signal velocity $v_{s i g}$ is equal to $N / T_{r}$. In this situation, after $t=T_{r}$ the last car moves faster than the leader. It gets ahead of its desired position and then tends to stand still again(or move very slowly). This 'stop-and-go' behavior continues until attenuation due to global stability sets in. The signal velocity is in terms of the number of cars per unit time. In the case that distances between successive cars are all equal to, say, $\Delta$, this is related to $v_{s}$, speed of sound, by $v_{s}=\Delta v_{\text {sig }}$.

This stop-and-go behavior can be seen from the above solution by neglecting the exponential decay. The motion of the trailing car then becomes (recall that a Galilean motion is of the form $x(t)=v_{0} t+x_{0}$ where $x_{0}$ and $v_{0}$ are constants.):

$$
x_{N}(t) \approx \text { Galilean }+\frac{4 v_{0}}{\pi \omega_{N}} \sum_{\ell=0}^{\infty}(-1)^{\ell+1} \frac{\sin \left((2 \ell+1) \omega_{N} t\right)}{(2 \ell+1)^{2}} .
$$

The periodic part of this motion is the sawtooth function (see Figure 5.2). The amplitude $A$ can be determined as follows: at $t=\frac{\pi}{2 \omega_{N}}$ each term $(-1)^{\ell+1} \sin \left((2 \ell+1) \omega_{N} t\right.$ is minimized and equal to -1 . Using that

$$
\sum_{\ell=0}^{\infty} \frac{-1}{(2 \ell+1)^{2}}=\frac{-\pi^{2}}{8}
$$

one obtains that $A=\frac{\sqrt{2} v_{0}}{|f|^{1 / 2}} N$. The velocity of $x_{N}(t)$ relative to the leader equals:

$$
\dot{x}_{N}(t)-v_{0} \approx \frac{4 v_{0}}{\pi} \sum_{\ell=0}^{\infty}(-1)^{\ell+1} \frac{\cos \left((2 \ell+1) \omega_{N} t\right)}{(2 \ell+1)} .
$$

This expression has an extremum at $t=0$ and so, since

$$
\sum_{\ell=0}^{\infty} \frac{(-1)^{\ell+1}}{(2 \ell+1)}=\frac{-4}{\pi}
$$


one obtains $\dot{x}_{N}(0)-v_{0}=-v_{0}$. Thus before attenuation kicks in, the last car will have a velocity that alternates between the values 0 and $2 v_{0}$, explaining the tendency for traffic to be stop-and-go, even though the leader happily sails along at constant velocity $v_{0}$, blissfully unaware - as (s)he receives no information - of the troubles others are having. This limit is not altogether physically reasonable, as clearly accelerations tend to become very large in the regions of the velocity changes.
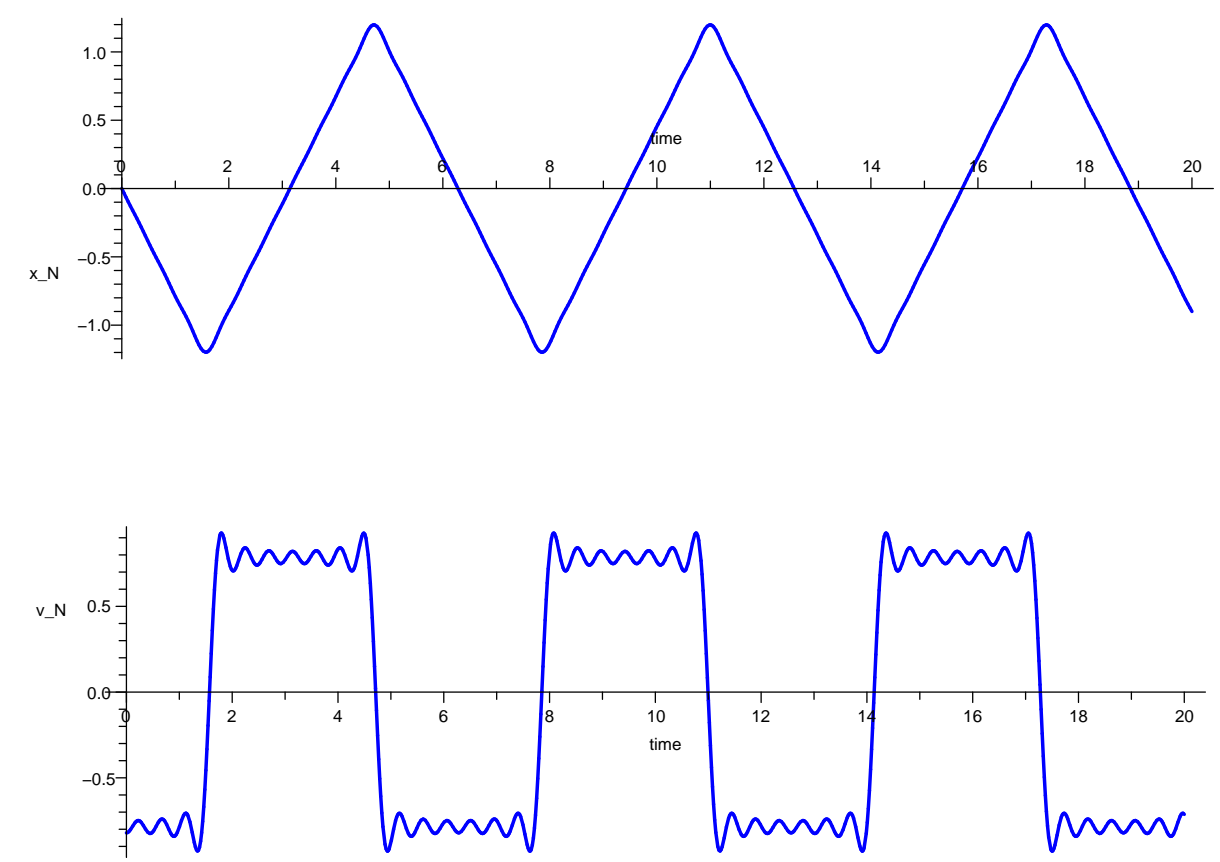

Figure 5.2: $\quad-\sum_{\ell \in \mathbb{N}}^{6} \frac{(-1)^{\ell} \sin \left((2 \ell+1) \omega_{N} t\right)}{(2 \ell+1)^{2}}$ and its derivative.

Exactly the same phenomena will occur when the flock travels at constant velocity and the leader decides to decelerate to a lower velocity from $v_{0}$ to $v_{0}-v_{1}$. Assume again that the distance between successive cars equals $\Delta$. It is easy to see that our model will then give rise to a wave of increasing amplitude traveling backwards from the leader with speed $v_{s}$ relative to the cars traveling at velocity $v_{0}$. The velocity of cars will tend to oscillate between $v_{0}-2 v_{1}$ and $v_{0}$. (If $v_{0}-2 v_{1}$ is negative, this prediction becomes unrealistic for actual traffic because cars will not acquire negative velocities.) If the distance between cars (in formation) is $\Delta$, the speed at which the perturbation propagates equals $v_{0}-\Delta \frac{\sqrt{|f|}}{\sqrt{2}}$.

If in the above scenario $v_{1}=v_{0}$ - the leader stops — and one imposes that the cars will stop as soon as their velocity has reached zero (and don't go in reverse), we get an estimate for the safe distance cars need to be traveling at, so that the leader can stop without causing collisions. Assume the cars were traveling in formation and thus that the distance between leader and N-th car was given by (see Equation (2.1)) $h_{0}-h_{N}$. Now suppose the leader starts breaking at $t=0$. Every-one has stopped at $t=T_{r}$ and

$$
x_{0}\left(T_{r}\right)-x_{N}\left(T_{r}\right)=h_{0}-h_{N}-A=h_{0}-h_{N}-\frac{\sqrt{2} v_{0}}{\sqrt{|f|}} N .
$$

If this number is positive, collisions can be avoided.

We summarize the main dynamical characteristics of this model in the Table below and comment on the design of an algorithm for the automated pilot. The symbols are explained in the text. Note that in Equation (2.1) we can rescale time so that the parameters $f$ and $g$ are replaced by -1 and $-|g| /|f|^{1 / 2}$. 


\begin{tabular}{|c||c|c|c|}
\hline Quantity & Symbol & Value Expressed in Parameters & Other Relations \\
\hline \hline Resonance Freq. & $\omega_{N}$ & $\frac{\pi \sqrt{|f|}}{2 \sqrt{2}} N^{-1}$ & $\omega_{N}=\Im \nu_{0}$ \\
\hline Freq. Resp. Fn. (for last car) & $a_{N}$ & - & $\begin{array}{c}\text { Peaks of Size } \propto N \text { at } \omega \propto \frac{1}{N} \\
\text { Exp. (in } N \text { ) Small elsewhere }\end{array}$ \\
\hline Peak Ampl of 1st Res & $a_{N}\left(\omega_{N}\right)$ & $\frac{8 \sqrt{2|f|}}{\pi^{2}|g|} N$ & See Theorem 3.3 \\
\hline Peak Ampl. Canon. Traffic Probl. & $A$ & $\frac{\sqrt{2} v_{0}}{\sqrt{|f|}} N$ & $A=\frac{\pi v_{0}}{2 \omega_{N}}$ \\
\hline Leading Lyapunov Expon. & $\Re \nu_{0}$ & $-\frac{\pi^{2}|g|}{16} N^{-2}$ & See Lemma 3.1 \\
\hline Response Time & $T_{r}$ & $\frac{\sqrt{2}}{\sqrt{|f|}} N$ & $T_{r}=\frac{A}{v_{0}}=\frac{\pi}{2 \omega_{N}}$ \\
\hline Signal Velocity & $v_{s i g}$ & $\frac{\sqrt{|f|}}{\sqrt{2}}$ & $v_{s i g}=\frac{N}{T_{r}}$ \\
\hline Speed of Sound & $v_{s}$ & $\left(h_{k-1}-h_{k}\right) \frac{\sqrt{|f|}}{\sqrt{2}}$ & $v_{s}=v_{s i g}$ (inter-car dist.) \\
\hline
\end{tabular}

The design of the algorithm consists in choosing the parameters. Note that the most obvious way to implement Equation (5.2) is to keep the desired distances $h_{i}-h_{i+1}$ (see Equation (2.1) between cars constant $(\Delta)$ and to require that this distance satisfies

$$
\Delta=h_{i}-h_{i+1}>\frac{\sqrt{2} v_{0}}{\sqrt{|f|}} .
$$

In the ideal design one would also want $A$ and $a_{N}\left(\omega_{N}\right)$ to be small (see Table). The role of $|f|$ here is rather complicated and the choice of its value might depend on what kind of perturbations the systems is expected to suffer. The role of $g$ is less complicated: it appears twice in the table and in both cases giving it a large value decreases oscillations.

By far the worst drawback of our linear design is the weak attenuation (for large $N$ ) of the oscillation due to the (leading) Lyapunov exponent $\Re \nu_{0}$ which is $\propto|g| N^{-2}$ (see Figure 5.3). This is ultimately due to the nature of the spectrum of $P$ or $L$ : the exponent equals the real part of the smallest eigenvalue of $P$.

\section{Appendix}

Lemma 6.1 For all $\epsilon>0$, there is $L>0$ so that, for large enough $N$, if $|\omega|>L N^{-1 / 2}$, then $\left|a_{N}(\omega)\right|<\epsilon$.

Proof: We need only check this for positive $\omega$. For small $\omega$ we have (see [8]) that there is $C>0$ such that

$$
\left|\mu_{+}(\omega)\right|=1+C \omega^{2}+\mathcal{O}\left(\omega^{4}\right) .
$$

We first prove that $\left|\mu_{+}(\omega)\right|$ is increasing for positive $\omega$. Since

$$
\partial_{\omega} \mu_{+}(\omega)=\frac{\partial_{\omega} \gamma}{\sqrt{\gamma^{2}-1}}\left(\gamma+\sqrt{\gamma^{2}-1}\right)
$$

one obtains

$$
\partial_{\omega} \ln \mu_{+}(\omega)=\frac{1}{f^{2}+\omega^{2} g^{2}} \frac{1}{\sqrt{2 f+\omega^{2}+2 i \omega g}}\left(2 f^{2}+\omega^{2} g^{2}-i \omega g f\right) .
$$

The argument of the left hand side can be seen to be in $\left(-\frac{\pi}{2}, \frac{\pi}{2}\right)$. This implies that $\Re \partial_{\omega} \ln \mu_{+}(\omega)>0$, and thus $\left|\mu_{+}(\omega)\right|$ is increasing. Now set $\omega>L N^{-1 / 2}$ and thus Lemma 3.2 implies that for an appropriate choice of $L$,

$$
\left|a_{N}(\omega)\right|<\frac{2}{e^{C L}-e^{-C L}}<\epsilon .
$$




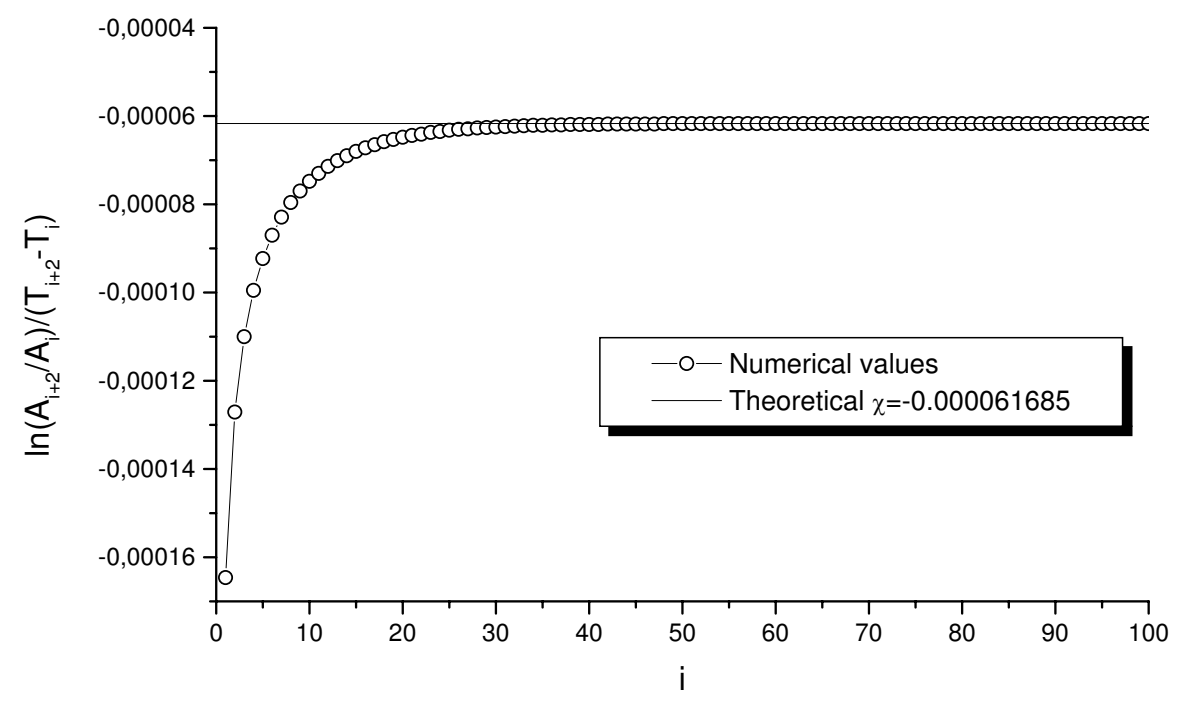

Figure 5.3: Calculation of the decay rate of the oscillation. The value converges to the predicted one $\Re \nu_{0}=6.2 \cdot 10^{-5}$ (here $f=g=-1$ ).

\section{References}

[1] R. Agaev, P. Chebotarev, On the spectra of nonsymmetric laplacian matrices, Linear Algebra App., 399:157168, 2005 .

[2] M. Bando, K. Hasebe, A. Nakayama, A. Shibata, Y. Sugiyama, Dynamical Model of Traffic Congestion and Numerical Simulation, Phys. Rev. E 51 1035-1042, 1995.

[3] John S. Caughman, G. Lafferriere, J. J. P. Veerman, A. Williams, Decentralized Control of Vehicle Formations, Systems \& Control Letters, 54, 899-910, 2005.

[4] John S. Caughman, J. J. P. Veerman, Kernels of Directed Graph Laplacians, Electr. J. Comb. 13, Vol 1, R39, 2006 .

[5] M. J. Lighthill, G.B. Whitham, Theory of Traffic Flow on Long Crowded Roads, Proc. Roy. Soc. Series A 229, $317-345,1955$

[6] J. J. P. Veerman, John S. Caughman, G. Lafferriere, A. Williams, Flocks and Formations, J. Stat.Phys. 121, Vol 5-6, 901-936, 2005.

[7] D.C. Gazis, R. Herman, R. W. Rothery, Nonlinear follow-the-leader models of traffic flow, Operations Res. 9 $1961545-567$.

[8] J. J. P. Veerman, B. D. Stosic, A. Olvera, Spatial Instabilities and Size Limitations of Flocks, Networks and Heterogneous Media 2, Vol 4, 647-660, 2007. 
[9] A. Williams, G. Lafferriere, J. J. P. Veerman, Stable Motions of Vehicle Formations, Proc. 44th Conference of Decision and Control, 72-77, 12-15 Dec. 2005.

[10] K. Nagel, M. Schreckenberg, A Cellular Automaton Model for Freeway Traffic, J. Phys. I France 2 (1992) 2221-2229.

[11] M. Treiber, A. Hennecke, D. Helbing, Congested traffic states in empirical observations and microscopic simulations, Phys. Rev E 62, Vol 2, 1805-1824, 2005.

[12] Some beautiful photographs of these phenomenona can be found in http://epod.usra.edu/archive/images/sortsolsum-05042006-hw.jpg

[13] G. L. Kotkin, V. G. Serbo, Problemas de Mecanica Clasica, Mir, Moscow, 1980. 